\title{
Hebb and the art of spine remodeling
}

\author{
Noam E Ziv ${ }^{1,2}$
}

Addresses: ${ }^{1}$ Department of Physiology \& Biophysics, Bruce Rappaport Faculty of Medicine, Technion - Israel Institute of Technology, 1 Efron Street, PO Box 9649, Haifa 31096, Israel; ${ }^{2}$ Network Biology Research Laboratories, Lorry Lokey Interdisciplinary Center for Life Sciences \& Engineering, Technion City, Haifa 32000, Israel

Email: noamz@netvision.net.il

Fl000 Biology Reports 2010, 2:69 (doi:10.3410/B2-69)

The electronic version of this article is the complete one and can be found at: http://f1000.com/reports/biology/content/2/69

\begin{abstract}
The notion that synaptic remodeling underlies certain forms of learning is one of the main tenets of Hebb's inspiring theories dating from the 1940s. Until recently, however, direct evidence for tight relationships between synaptic remodeling and behavior has been scarce. Fascinating data from recent studies on the remodeling of postsynaptic structures known as dendritic spines indicates that such relationships might be more complex than initially expected.
\end{abstract}

\section{Introduction and context}

At the most basic level, the brain may be viewed as a vast network of nerve cells (neurons) with specialized projections (dendrites and axons) connected to each other at junctions known as synapses, through which signals are passed from one neuron to the next. As early as the $19^{\text {th }}$ century, well before the exact nature of signals conveyed by neurons and synapses was understood, changes in the ability of one neuron to 'drive' another were hypothesized to underlie the formation of new associations and the learning of new tasks $[1,2]$. Years later, Donald Hebb rephrased these ideas in his influential monograph Organization of Behavior [3], suggesting that when one neuron repeatedly takes part in driving a second neuron "some growth process or metabolic change takes place" at synapses that increases the efficiency of the first neuron in driving the second [3].

Inspired by Hebb's writings, countless attempts have been made to uncover the nature of these 'growth processes' and how these might relate to learning. Much of this work has focused on dendritic spines; the tiny 'door-knob' shaped protrusions that extend from the dendrites' shafts, mainly because these are the postsynaptic components of most excitatory synapses in the mammalian central nervous system (CNS). As it is commonly assumed that persistent changes in synaptic connectivity ultimately require structural changes, many studies have focused on the structural remodeling of dendritic spines, or more specifically, on changes in spine numbers, densities, sizes, and shapes. Only during the last decade, however, with the development of tools for long-term imaging of individual spines in intact brains, have these ideas been directly addressed. This commentary will focus on a subset of studies published over the last two years. For more comprehensive reviews, see [4-6].

\section{Major recent advances}

Imaging of dendritic spines over many days and weeks in adult animals (mainly mice) has revealed that the majority of dendritic spines are quite persistent (reviewed in [4-6]). Yet, these studies also reported that new dendritic spines form and others disappear at rates of a few percent per day. Furthermore, experimental manipulations that caused partial deprivation of sensory input (trimming certain whiskers or monocular deprivation) were shown to significantly affect various aspects of spine remodeling [4-8]. Importantly, these changes were found to correlate, in some cases, with functional changes in the imaged neurons. For example, in the binocular region of the mouse, monocular deprivation was shown to be associated with both increased 
responsiveness to light in the other (spared) eye and increases in the number of dendritic spines of layer 5 pyramidal neurons within the same region [7]. Similarly, in mouse cortical regions that respond equally to neighboring whiskers, whisker trimming in a 'checkerboard' pattern was associated with both increased responsiveness to the spared whisker and an increase in the fraction of new spines of layer 5 pyramidal neurons that became persistent rather than disappearing [8].

Other recent studies have gone further and directly examined relationships between spine remodeling and behavioral learning. In two such studies, mice were trained to perform new motor tasks (e.g., single seed reaching [9] or remaining on an accelerating rotating rod [10]) and (two-photon) microscopy was used to examine if learning the skill was associated with changes in dendritic spine remodeling not seen in untrained mice. Both studies revealed that by the end of the first 1-2 days of training, the number of new spines in particular brain regions had nearly doubled in comparison with untrained mice. However, continuous training was subsequently followed by increased rates of spine elimination, and after 1-2 weeks, total dendritic spine numbers did not differ between trained and untrained animals. Remarkably, amongst trained animals behavioral performance correlated well with the numbers of new spines formed shortly after training (and with the extent of pre-existing spine elimination). In both studies it was shown that training-associated changes in dendritic spine remodeling were regionally specific, with changes confined to specific cerebral cortex regions concerned with limb movement. However, similar degrees of spine remodeling could be induced in cortical regions concerned with sensory input (from whiskers) in mice exposed to enriched environments [10].

A common finding in many of these studies is that the experimental manipulations used did not seem to increase the rate at which transient spine precursors were generated. Instead, they seemed to increase the fraction of new spines that became persistent $[8,9,11]$, as if learning a new task or adapting to altered sensory input favors the stabilization of particular transient spines associated with that function $[4,12,13]$. This feature is most strongly illustrated in a recent study performed in juvenile zebra finches as they learn to imitate a 'tutor' song [14]. Here it was shown that (a) higher levels of spine turnover were correlated with a greater capacity for song imitation, and (b) hearing the tutors' song led to dendritic spine stabilization and enlargement that was associated with enhanced synaptic activity in the corresponding brain regions.
A fascinating facet of these studies is the observation that later re-exposure of trained animals to the previously learned task $[9,10]$ or the same sensory deprivation [7] did not lead to any particular dendritic spine remodeling. On the other hand, spine remodeling processes similar to those observed during acquisition of the original tasks were observed again when the animals learned a new motor task.

\section{Future directions}

The aforementioned studies seem to indicate that a strong correspondence exists between sensory adaptation or task learning and spine remodeling. So what is wrong with this picture?

An implicit, somewhat naïve perhaps, interpretation of the aforementioned studies is that the dendritic spines that appear following experimental manipulations represent newly formed connections between functionally relevant neurons and the imaged neuron and that these synapses embody stronger connections between these neurons. For example, in the case of monocular deprivation, it might be surmised that the new dendritic spines mediate additional connections from neurons carrying information from the spared eye and thus enhance the input from that eye. In support of this idea, electron-microscope-based analysis indicates that new spines can form additional connections with the same presynaptic neurons [15]. This interpretation thus implies that the new connections become major determinants of altered postsynaptic functionality, if not immediately, then with time, as part of a process often referred to as 'consolidation'.

The problem with this interpretation is that the very same studies show that the correspondence between spine remodeling and functional changes (sensory adaptation or task learning) is not complete. For example, while restoration of binocular vision after monocular deprivation is followed by complete recovery of function in the binocular region, the new spines that formed during the deprivation period are not eliminated [7]. Furthermore, a second round of monocular deprivation does not lead to a second wave of spine formation even though functionally, responses to the spared eye are enhanced again (and even more than the first time) [7]. Finally, while structural remodeling is observed in corresponding layer 5 pyramidal neurons in the cerebral cortex, it is not observed for layer 3 neurons despite the fact that these neurons exhibit robust functional ocular dominance shifts [7]. Similarly, while measured responses to spared whiskers correlate well with increased dendritic spine persistence in some (the septal) regions of the corresponding cerebral cortex, in adjacent 
regions, no particular spine remodeling was observed even though responses to trimmed whisker deflection dropped by $68 \%(!)[8]$.

One possible and quite straightforward explanation for the incomplete correspondence between spine remodeling and neuronal function is that it reflects experimental constraints. In all these studies, imaging was limited to the most superficial layers of the cortex. This leaves open the possibility that spine remodeling recorded in superficial layers is merely a faint echo of much more vigorous dendritic spine remodeling occurring in deeper layers (e.g., in layer 4, where major thalamocortical inputs terminate [16]).

If this is not the case, however, one is left to conclude that major changes in functionality can occur without corresponding changes in dendritic spine turnover or spine numbers in the same region. Perhaps there are structural changes but they occur in other brain regions. Alternatively, structural changes might occur at inhibitory synapses, which typically do not form on dendritic spines and thus would be missed. Finally, even though pre- and postsynaptic structural measures typically correspond well with each other [4], structural remodeling may be, in some cases, more prominent presynaptically $[17,18]$ (reviewed in [19]). Presynaptic remodeling is particular noteworthy here because Hebb originally suggested that synaptic remodeling occurs in presynaptic 'knobs' (not dendritic spines), which he describes as "rather irregular thickening[s] in the unmyelinated part of an axon near its ending, where it is threading its way through a thicket of dendrites and cell bodies" [3]. Hebb writes: "When one cell repeatedly assists in firing another, the axon of the first cell develops synaptic knobs (or enlarges them if they already exist) in contact with the soma of the second cell" (note that in Hebb's monograph "... 'Soma' refers to dendrites and body or all of the cell except the axon" [3]).

At present, there is no conclusive information that unequivocally supports or refutes any of these alternative types of structural changes. Thus, it remains possible that the naïve interpretation is wrong. Perhaps the observed structural changes do not necessary involve the 'immediate upstream suspects' (which would be, in the case of monocular deprivation for example, neurons carrying information from the spared eye) and are instead part of more complex reconfigurations of distributed networks, akin to the 'cell assemblies' postulated by Hebb [3]. Alternatively, perhaps, 'Lady Macbeth's argument' [20], that "what is done cannot be undone" holds true here and that once structural changes occur, they are not rapidly reversed after the forces that induced them are removed [7]. In fact, Hebb writes on this matter: "... Cajal (among others) conjectured that the change at the synapse in learning is an amoeboid outgrowth of the cell which might need very little time for its occurrence". Hebb then speculates: "An amoeboid outgrowth would be reversible; but the mere absence of the electrochemical influence which produced the outgrowth might not act as forcefully and promptly as its presence. So it is possible to assume ... that synaptic decay occurs slowly and perhaps is never quite complete". Finally, a glum possibility that cannot yet be disregarded is that spine formation and elimination in the experiments described above are merely by-products of little functional significance that appear in the wake of other changes of greater functional importance that do not happen to be under the proverbial lamppost in imaging-based experiments.

Hebb points to an alternative to new synapse formation, that is, a change in the size of existing synapses: "the greater the area of contact the greater the likelihood that action in one cell will be decisive in firing another" [3]. Indeed, in some of the aforementioned studies $[7,11,14]$ (but see [9]) changes in dendritic spine head size were observed to correlate well with functional changes in the imaged tissue. As dendritic spine head size and synaptic strength show a direct correlation (reviewed in [4]), perhaps spine size rather than spine addition and removal is a more functionally relevant measure of dendritic spine remodeling. Yet this form of remodeling is not entirely without problems either. Firstly, spine head size tends to change not only in response to specific activity patterns but also spontaneously, even when activity is suppressed or eliminated entirely [21,22]. Such spontaneous remodeling, acting over long-enough time scales, could drown functionally relevant spine remodeling events in a sea of functionally irrelevant ones [22]. Secondly, spine size is also sensitive to overall activity levels [22] - a factor that cannot be ignored in the interpretation of data obtained in sensory deprivation paradigms. Finally, even changes in dendritic spine size directly induced by acute, specific activity patterns in an in vitro preparation have been shown to gradually revert over a few days [11].

So what are we left to conclude? The old notion that synaptic remodeling, and in particular spine remodeling, underlies important forms of adaptation and learning has been greatly substantiated by the elegant experimental approaches developed over the last decade. Yet the same approaches have also pointed to puzzling discrepancies that have yet to be resolved. Perhaps further development of tools that would allow for 
long-term imaging of both pre- and post-specializations, inhibitory and excitatory synapses, and at all cortical layers will resolve some of these discrepancies. Alternatively, it might be prudent to listen carefully to Hebb's words on two crucial issues: the uniqueness of synapses and the importance of population thinking. Starting with the former, Hebb undoubtedly favored synapses as loci for changing connectivity within cell assemblies. Yet he also writes: "...there is certainly no direct evidence that this is so and the postulated change if it exists may be metabolic, affecting cellular rhythmicity and limen [i.e., threshold]"; on the latter Hebb writes: "At each synapse there must be a considerable dispersion in the time of arrival of impulses, and in each individual fiber a constant variation of responsiveness; and one could never predicate a determinate pattern of action in any small segment of the system. In the larger system, however, a statistical constancy might be quite predictable".

In this spirit, the prevailing view of neurons as historyindependent 'point functions' connected to each other through unique, history-dependent and dynamic synapses should, perhaps, be expanded to a more inclusive view that considers large populations of synapses and neurons with rich history dependencies at multiple levels and time scales [23], where structural changes in dendritic spines are only one of many types of changes that take place during adaptation and learning processes [24-27]. Given his inspiring concepts concerning cell assemblies and their statistical properties, Hebb would probably agree.

\section{Abbreviations}

CNS; central nervous system.

\section{Competing interests}

The author declares that he has no competing interests.

\section{Acknowledgments}

My apologies to the authors of numerous studies that could not be mentioned due to space limitations. This report was supported by grants from the Israel Science Foundation (526/01, 1780/07) and the United States Israel Binational Science Foundation (2007425).

\section{References}

I. James W: Chapter XIV: Association. In The Principles of Psychology. Volume 2. New York: Henry Holt and Company; 1890.

2. Freud S: Project for a Scientific Psychology. In The Standard Edition of the Complete Psychological Works of Sigmund Freud, Volume I (I886-1899): Pre-Psycho-Analytic Publications and Unpublished Drafts. London: Hogarth Press; 1953 (1895).

3. Hebb DO: Organization of Behavior: A Neuropsychological Theory. New York: John Wiley and Sons; 1949.
4. Holtmaat A, Svoboda K: Experience-dependent structural synaptic plasticity in the mammalian brain. Nat Rev Neurosci 2009, 10:647-58

5. Bhatt DH, Zhang S, Gan WB: Dendritic spine dynamics. Annu Rev Physiol 2009, 7I:26I-82.

6. Alvarez VA, Sabatini BL: Anatomical and physiological plasticity of dendritic spines. Annu Rev Neurosci 2007, 30:79-97.

7. Hofer SB, Mrsic-Flogel TD, Bonhoeffer T, Hübener M: Experience leaves a lasting structural trace in cortical circuits. Nature $2009,457: 313-17$

FI000 Factor 6.4 Must Read Evaluated by Elizabeth Gould 03 Dec 2008, Brett Adams 29 Jan 2009

8. Wilbrecht L, Holtmaat A, Wright N, Fox K, Svoboda K: Structural plasticity underlies experience-dependent functional plasticity of cortical circuits. J Neurosci 2010, 30:4927-32.

9. Xu T, Yu X, Perlik AJ, Tobin WF, Zweig JA, Tennant K, Jones T, Zuo $Y$ : Rapid formation and selective stabilization of synapses for enduring motor memories. Nature 2009, 462:915-9.

10. Yang G, Pan F, Gan WB: Stably maintained dendritic spines are associated with lifelong memories. Nature 2009, 462:920-4.

FI000 Factor 6.5 Must Read

Evaluated by Ted Dawson 08 Jan 2010, Hisashi Umemori 18 Jan 2010, John Lisman 29 Jan 2010

II. De Roo M, Klauser P, Muller D: LTP promotes a selective longterm stabilization and clustering of dendritic spines. PLOS Biol 2008, 6:e219.

FI000 Factor 4.8 Must Read

Evaluated by Daniel Madison 24 Oct 2008, John Lisman 3I Dec 2009

12. Jontes JD, Smith SJ: Filopodia, spines, and the generation of synaptic diversity. Neuron 2000, 27: I I-4.

13. Ziv NE, Ahissar E: New tricks and old spines. Nature 2009, 462:859-61.

14. Roberts TF, Tschida KA, Klein ME, Mooney R: Rapid spine stabilization and synaptic enhancement at the onset of behavioural learning. Nature 2010, 463:948-52.

15. Knott GW, Holtmaat A, Wilbrecht L, Welker E, Svoboda K: Spine growth precedes synapse formation in the adult neocortex in vivo. Nat Neurosci 2006, 9: I I 17-24.

FI000 Factor 4.8 Must Read

Evaluated by Noam Ziv 18 Aug 2006, Thomas Biederer 19 Sep 2006

16. Coleman JE, Nahmani M, Gavornik JP, Haslinger R, Heynen AJ, Erisir A, Bear MF: Rapid structural remodeling of thalamocortical synapses parallels experience-dependent functional plasticity in mouse primary visual cortex. I Neurosci 2010, 30:9670-82.

17. Becker N, Wierenga C], Fonseca R, Bonhoeffer T, Nägerl UV: LTD induction causes morphological changes of presynaptic boutons and reduces their contacts with spines. Neuron 2008, 60:590-7.

18. Matz J, Gilyan A, Kolar A, McCarvill T, Krueger SR: Rapid structural alterations of the active zone lead to sustained changes in neurotransmitter release. Proc Natl Acad Sci U S A 2010, 107:8836-4l.

19. Krueger S, Fitzsimonds RM: Remodeling the plasticity debate: the presynaptic locus revisited. Physiology (Bethesda) 2006, 21:346-51.

20. Dudai $Y$ : The neurobiology of consolidations, or, how stable is the engram? Annu Rev Psychol 2004, 55:5I-86.

21. Yasumatsu N, Matsuzaki M, Miyazaki T, Noguchi J, Kasai $\mathrm{H}$ : Principles of long-term dynamics of dendritic spines. J Neurosci 2008, 28: 13592-608. 
22. Minerbi A, Kahana R, Goldfeld L, Kaufman M, Marom S, Ziv NE: Long-term relationships between synaptic tenacity, synaptic remodeling, and network activity. PLoS Biol 2009, 7:el000I36.

FI000 Factor 9.0 Exceptional

Evaluated by Timothy Ryan 08 Jul 2009

23. Marom S: Neural timescales or lack thereof. Prog Neurobiol 2010, 90:16-28.

24. Marder E, Abbott LF, Turrigiano GG, Liu Z, Golowasch J: Memory from the dynamics of intrinsic membrane currents. Proc Natl Acad Sci U S A 1996, 93:1348I-6.
25. Zhang W, Linden DJ: The other side of the engram: experiencedriven changes in neuronal intrinsic excitability. Nat Rev Neurosci 2003, 4:885-900.

26. Daoudal G, Debanne D: Long-term plasticity of intrinsic excitability: learning rules and mechanisms. Learn Mem 2003 , 10:456-65.

27. Mozzachiodi R, Byrne JH: More than synaptic plasticity: role of nonsynaptic plasticity in learning and memory. Trends Neurosci 2010, 33:17-26.

FI000 Factor 6.0 Must Read

Evaluated by Randolf Menzel 19 Jan 2010 